\title{
The peptide nucleic acids (PNAs), powerful tools for molecular genetics and cytogenetics
}

\author{
Franck Pellestor ${ }^{*}$ and Petra Paulasova ${ }^{2}$ \\ ${ }^{1}$ CNRS UPR 1142, Institute of Human Genetics, 141 rue de la Cardonille, F-34396 Montpellier Cedex 5, France; \\ ${ }^{2}$ Centre of Assisted Reproduction and Reproductive Genetics, Institut of Biology and Medical Genetics, Motol Hospital, \\ V uvalu 84, 15006 Praha 5, Czech Republic
}

Peptide nucleic acids (PNAs) are synthetic mimics of DNA in which the deoxyribose phosphate backbone is replaced by a pseudo-peptide polymer to which the nucleobases are linked. PNAs hybridize with complementary DNAs or RNAs with remarkably high affinity and specificity, essentially because of their uncharged and flexible polyamide backbone. The unique physico-chemical properties of PNAs have led to the development of a variety of research assays, and over the last few years, the use of PNAs has proven their powerful usefulness in molecular biology procedures and diagnostic assays. The more recent applications of PNA involve their use as molecular hybridization probes. Thus, several sensitive and robust PNA-dependent methods have been designed for developing antigene and anticancer drugs, modulating PCR reactions, detecting genomic mutation or labelling chromosomes in situ.

European Journal of Human Genetics (2004) 12, 694-700. doi:10.1038/sj.ejhg.5201226

Published online 23 June 2004

Keywords: PNA; PNA/DNA duplex; mutation detection; PNA-PCR; PNA-FISH

\section{Introduction}

Advances in genomic technologies have opened up new avenues in the diagnostics and the management of human diseases. In genetics, the advent of molecular techniques has brought forth new procedures for increasingly specific, rapid and reliable diagnostic and predictive DNA analysis. In this way, development of nucleic acid analogs has become an important feature because of the potential use of these new biomarkers in genetic diagnostics and investigations. Over the last decade, nucleic acids have been extensively modified, by replacing the posphodiester linker or the sugar-phosphodiester backbone by various neutral or charged structures. Several of these modified oligonucleotides have improved properties in terms of affinity and binding to DNA and RNA. Two significant representatives of this new type of oligomers are locked

*Correspondence: Dr F Pellestor, CNRS UPR 1142, Institute of Human Genetics, 141 rue de la Cardonille, F-34396 Montpellier Cedex 5, France. Tel: + 3349961 9912; Fax: + 334996199 01;

E-mail: franck.pellestor@igh.cnrs.fr

Received 30 January 2004; revised 13 April 2004; accepted 23 April 2004 nucleic acids (LNAs), also known as bridged nucleic acids (BNAs), and peptide nucleic acids (PNAs).

LNAs consist of monomer nucleotides connected by a $2^{\prime}-4^{\prime}$ methylene bridge. The entropic constraint imposed by the $2^{\prime}-4^{\prime}$ linker increases the binding affinity of LNAs for complementary nucleic acids. In addition, LNAs display an enhanced resistance to nuclease degradation. As LNAs possess a phosphodiester backbone, they have a good aqueous solubility and can be introduced into cells by standard transfection techniques. Thus, these molecules could act as antiproliferative agents, but further studies are needed to exactly determine the pharmacokinetic properties of LNAs.

Among all the modified oligonucleotides designed, the PNAs constitute a remarkable class of nucleic acid mimics, with important physico-chemical properties that have been exploited to develop a wide range of powerful biomolecular tools, including molecular probes, biosensors and antigene agents. The present paper provides an overview of PNAs properties and the techniques exploiting PNA technology in molecular genetics and cytogenetics. 


\section{PNA structure and properties}

The PNAs have been introduced by Nielsen et al. ${ }^{1}$ PNAs are synthetic DNA analogs in which the phosphodiester backbone is replaced by repetitive units of $\mathrm{N}$-(2-aminoethyl) glycine to which the purine and pyrimidine bases are attached via a methyl carbonyl linker (Figure 1). The procedures for PNA synthesis are similar to those employed for peptide synthesis, using standard solid-phase manual or automated synthesis. The PNA molecules can routinely be labelled with biotin or fluorophores. A subsequent generation of PNAs could involve modification of the $\mathrm{N}$-(2aminoethyl) glycine backbone (PNA analogs) or chimeric architecture, like PNA-peptide chimeras or PNA-DNA chimeras developed in order to improve the solubility and the cellular uptake of PNAs or to exhibit new biological properties. $^{2}$ The synthetic backbone provides PNA with unique hybridization characteristics. Unlike DNA and RNA, the PNA backbone is not charged. Consequently, there is no electrostatic repulsion when PNAs hybridize to its target nucleic acid sequence, giving a higher stability to the PNA-DNA or PNA-RNA duplexes than the natural homo- or heteroduplexes. This greater stability is reflected by a higher thermal melting temperature $\left(T_{\mathrm{m}}\right)$, as compared to the corresponding DNA-DNA or DNA-RNA duplexes. $^{3}$

An additional consequence of the polyamide backbone is that PNAs hybridize virtually independently of the salt concentration. Thus, the $T_{\mathrm{m}}$ of PNA-DNA duplex is barely affected by low ionic strength. This property can be exploited when targeting DNA or RNA sequences involved in secondary structures, which are destabilized by low ionic strength. This facilitates the hybridization with the PNAs. The unnatural backbone of PNAs also means that PNAs are not degraded by nucleases or proteases. For instance, incubation of PNAs with S1 nuclease or DNAse I has no effect on PNA. ${ }^{4}$ Owing to this resistance to the enzyme degradation, the lifetime of PNAs is extended both in vivo and in vitro. Also, PNAs are not recognized by polymerases and therefore cannot be directly used as primers or be copied.

PNAs hybridize to complementary DNA or RNA in a sequence-dependent manner, according to the WatsonCrick hydrogen bonding scheme. In contrast to DNA, PNA can bind in either parallel or antiparallel manner. However, the antiparallel binding is favoured over the parallel one. Structural information on the PNA binding modes have been obtained by nuclear magnetic resonance and by X-ray crystallography. ${ }^{5}$ PNA are able to adopt both A- and B-type structures when associating with RNA and DNA, respectively, whereas PNA-PNA duplexes form an unusual helix conformation, called P-type and are characterized by a large pitch of 18 base pairs. ${ }^{6}$

PNA probes can bind to either single-stranded DNA or RNA, or to double-stranded DNA (Figure 2). Homopyrimidine PNAs with a minimum of 10-mers, as well as PNAs
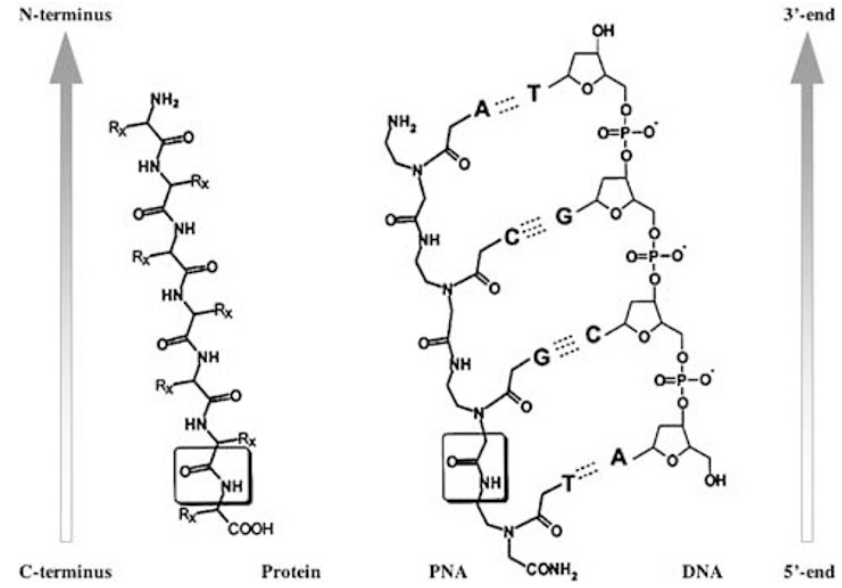

Figure 1 Chemical structures of PNA as compared to DNA and protein. The backbone of PNA displays 2aminoethyl glycine linkages in place of the regular phosphodiester backbone of DNA, and the nucleotides bases are attached to this backbone at the amino-nitrogens through methylene carbonyl linkages. The amide bond characteristic for both PNA and protein is boxed in. By convention, PNAs are depicted like peptides, with the $\mathrm{N}$-terminus at the left (or at the top) position and the c-terminus at the right (or at the bottom) position. PNAs hybridise to complementary DNA or RNA sequences in a sequence-dependent manner, following the Watson-Crick hydrogen bonding scheme. PNAs can bind to complementary nucleic acids in both parallel and antiparallel orientation. However, the antiparallel orientation illustrated in this figure is preferred. The Watson-Crick hydrogen bonds are indicated by '.....'.

containing a high proportion of pyrimidine residues, bind to complementary DNA sequences to form highly stable (PNA) $)_{2}$-DNA triplex helices displaying $T_{\mathrm{m}}$ over $70^{\circ} \mathrm{C}$. In these triplexes, one PNA strand hybridizes to DNA through standard Watson-Crick base pairing rules, while the other PNA strand binds to DNA through Hoogsteen hydrogen bonds. The resulting structure is called P-loops. The stability of these triple helixes is so high that homopyrimidine PNA targeted to purine tracts of dsDNA invades the duplex by displacing one of the DNA strands. ${ }^{6}$ The efficiency of this strand invasion can be further enhanced by using two homopyrimidine PNA oligomers connected via a flexible linker or by the presence of nonstandard nucleobases in the PNA molecule.

Finally, PNA-DNA hybridization is significantly more affected by base mismatches than DNA-DNA hybridization. Using a 15-mer PNA, all possible single mismatch combinations were tested in both PNA/DNA duplexes and corresponding DNA/DNA duplexes. In the PNA/DNA duplexes, the average $T_{\mathrm{m}}$ was $15^{\circ} \mathrm{C}$, whereas it was $11^{\circ} \mathrm{C}$ in the corresponding DNA/DNA duplexes. ${ }^{6,7}$ Similar results were obtained for PNA/RNA duplexes. ${ }^{3}$ This high level of discrimination at single base level indicates that short PNA 
1

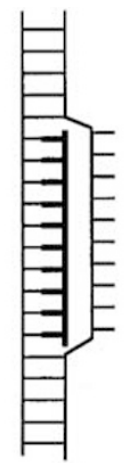

Duplex invasion
2

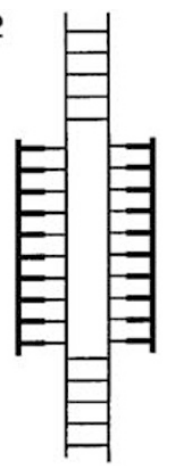

Double duplex invasion
3

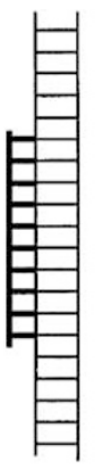

Triplex
4

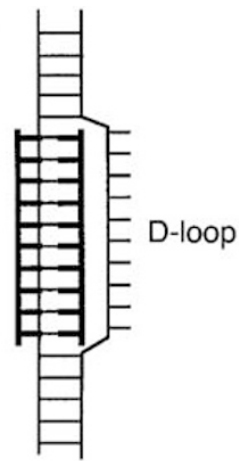

Triplex invasion

Figure 2 Schema of PNA binding modes for targeting double-stranded DNA. PNA oligomers are drawn in bold. (1) Standard duplex invasion complex formed with some homopurine PNAs. (2) Double-duplex invasion complex, very stable but only possible with PNAs containing modified nucleobases. (3) Conventional triple helical structure (triplex) formed with cytosinerich homopyrimidine PNAs binding to complementary homopurine DNA targets. (4) Stable triplex invasion complex, leading to the displacement of the second DNA strand into a 'D-loop'.

probes could offer high specificity, and thus allow the further development of several PNA-based strategies for molecular investigations and diagnosis.

\section{Applications of PNA technology}

PNAs are used as powerful tools not only in research laboratories but also in prognostics, diagnostics and disease monitoring in molecular medicine. Since its introduction, an increasing number of applications of PNA technology have been described, confirming the high potential of PNAs in molecular genetic investigations.

\section{Antisense and antigene applications}

Originally conceived as agents for double-stranded DNA binding, the unique properties of PNAs as DNA mimics were first exploited for gene therapy drug design. PNAs can inhibit transcription and translation of genes by tight binding to DNA or mRNA.

PNA-mediated inhibition of gene transcription is mainly due to the formation of strand invaded complexes or strand displacement in a DNA target. Several in vitro studies have shown that the binding of PNA or bis-PNA to complementary DNA can efficiently block transcriptional elongation and inhibit the binding of transcriptional factors. Thus, Boffa et $a l^{8}$ reported that PNA invasion of the tandem CAG repeat of the human androgen receptor and the TATA binding protein inhibits the transcription of these genes. PNA-mediated inhibition of transcription was also demonstrated for the alpha-chain of the interleukin-2 receptor. ${ }^{9}$ PNA targeted against the promoter region of a gene can form stable PNA-DNA complexes that restrict the DNA access of the polymerase, whereas PNA complexes located far from the promoter can block the polymerase progression and lead to the production of truncated RNA transcripts. Nielsen et al ${ }^{10}$ have demonstrated that even an 8-mer PNA can efficiently block transcriptional elongation by $(\mathrm{PNA})_{2}-\mathrm{DNA}$ triplex formation. Another way to alter gene transcription is based on the use of PNAs as competitors with endogenous cis-element(s) present in the target gene for trans-acting factors. This results in an attenuation of the authentic interactions of trans-factors with their cis-elements. ${ }^{11}$

PNAs are able to interact with mRNA independently of the RNA secondary structure. Studies on the mechanisms of antisense activity have demonstrated that PNA inhibits expression differently than antisense oligonucleotides acting through RNase-H mediated degradation of the mRNA-oligonucleotide hybrid. Since PNAs are not substrates for RNAse, their antisense effect acts through steric interference of either RNA processing, transport into cytoplasm or translation, caused by binding to the mRNA. $^{12}$

Application of PNAs as antisense reagents was first demonstrated in 1992. The nuclear microinjection of a 15-mer PNA targeting the translation start region of SV40 large $\mathrm{T}$ antigen mRNA inhibited transcription in cell extracts. ${ }^{13}$ This inhibition was both sequence-specific and dose-dependent. More recently, Mologni et al ${ }^{14}$ reported the effect of three different types of antisense PNAs on the in vitro expression of the PML/RARalpha gene. The PNAs used targeted various sites involving translocation start sites, coding sequences and the 5'-untranslated region (UTR). It was also reported that intron-exon splice junctions were very sensitive targets for PNA antisense probes because correct mRNA splicing can be altered by PNA binding. ${ }^{15}$

The good stability of PNA oligomers, their strong binding efficiency and their lack of toxicity at even relatively high concentrations suggests that PNAs could constitute highly efficient compounds for effective antisense/antigene application. However, despite the initial 
rapid success of PNA-based approaches in vitro, progress in the use of PNAs as tools for regulating gene expression was hampered by the slow cellular uptake of 'naked' PNAs by living cells. Subsequent modifications of PNAs have led to significant improvements in the uptake of PNA in eukaryotic cells. Delivery into the cell can be speeded up by coupling PNA to DNA oligomers, to receptor ligands or more efficiently to peptides such as cell penetrating peptides which are rapidly internalized by mammalian cells. ${ }^{16}$ Several reports have demonstrated that PNAs conjugated to such peptides are efficiently taken up by eukaryotic cells. Pooga et $a^{17}$ reported the use of a PNApeptide conjugate in the downregulation of the galanin receptor in neuronal cells in culture. The intrathecal injection of the PNA conjugate into the brain of living rats induced attenuated receptor activity in the rat brain. ${ }^{18}$ Another strategy adapted to improve the in vivo delivery of PNA can be their incorporation into liposomes. ${ }^{19}$

Thus, the in vitro cellular delivery of PNA is the major challenge that must be overcome before it can be efficiently used as a therapeutic agent. Results of the various proposed methods to facilitate the cell delivery of PNAs are promising and indicate that PNAS guided by suitable vectors can work as chemotherapeutic agents.

PNA-peptide duplexes, which can penetrate into cells, have been used in anticancer applications. In this manner, telomerase activity in human melanoma cells and tumour specimens was inhibited by PNA conjugated with Antennapedia derived peptide (Antp) at nanomolar concentrations. ${ }^{20}$ Since telomerase is almost ubiquitously expressed in human tumours, these data point out the potential use of PNAs as anticancer drugs.

\section{PNA as delivery agent}

A major limitation of nonviral gene therapy is the low efficiency of gene transfer into target cells. PNAs can be use as adapters to link peptides, drugs or molecular tracers to plasmid vectors. According to the binding site, the coupling of PNAs to plasmids has no effect either on the transcription of genes included in the plasmid or on the plasmid's physiological activities. Thus, this approach allows circumvention of such barriers to gene transfer and fixation of drugs to plasmids in order to enhance the gene delivery or tissue-specific targeting. ${ }^{21}$ Using a triplex forming PNA as linker, Braden et $a l^{22}$ observed an eight times higher nuclear localization of a coupled nuclear localization signal (NLS) than did the free oligonucleotide.

\section{PCR and Q-PNA PCR}

PNA probes have no direct interaction with DNA polymerase but PNAs can terminate the elongation of oligonucleotide primers by binding to the template or competing with the primers. ${ }^{23}$ Moreover, PNA-DNA chimeras can be recognized by the DNA polymerase and can thus be used as primers for PCR reactions. ${ }^{24}$ The high affinity binding of
PNAs has also been used for detecting single base pair mutations by PCR. This strategy, named PNA directed PCR clamping, uses PNAs to inhibit the amplification of a specific target by direct competition of the PNA targeted against one of the PCR primer sites and the conventional PCR primer. ${ }^{25}$ This PNA-DNA complex formed at one of the primer sites effectively blocks the formation of the PCR product. The procedure is so powerful that it can be used to detect single base-pair gene variants for mutation screening and gene isolation. ${ }^{26}$

More recently, novel automated realtime PCR has been developed using PNAs. In this method, named Q-PNA PCR, a generic quencher labelled PNA (Q-PNA) is hybridized to the $5^{\prime}$ tag sequence of a fluorescent dye-labelled DNA primer in order to quench the fluorescence of the primer. During PCR, the Q-PNA is displaced by incorporation of the primer into amplicons and the fluorescence of the dye label is liberated. ${ }^{27}$

Furthermore, LightUp probe and LightSpeed probe technologies have taken advantage of PNA properties. LightUp probes consist of a thiazole orange conjugated with a PNA oligomer, whereas LightSpeed probes are duallabelled PNAs with a fluorescent dye and a quencher dye bound to the opposing termini of the molecule. ${ }^{28,29}$ Both LightUp and LightSpeed probes are unique reagents for real-time PCR, which combine fluorescent enhancement with the high sensitivity and specificity of PNAs. A singlebase mismatch in the target sequence is sufficient to prevent probe binding. ${ }^{30}$

\section{Nuclear acid capture}

PNAs can be used for sequence-specific capture of singlestranded nucleic acids taking advantages of the tight complex formation at low ionic strength, which destabilizes nucleic acid secondary structure. Several studies have reported such applications of PNAs. Short PNA probes can then be used as generic capture probes for purification of nucleic acids. A system for capture of double-stranded DNA was also experimented using (PNA) $)_{2}$-DNA openers creating a large single-strand DNA loop to which a biotinylated oligonucleotide can hybridize. This complex allows the capture of the DNA via streptavidin beads. ${ }^{31}$ Analogous to the padlock hybrydization system, ${ }^{32}$ such a procedure may be used for detecting specific DNA sequence, for example on chromosomes, by enzymatic primer extension of the bound oligonucleotide. Also, the ability of pyrimidine PNAs to serve as opener for specific DNA sites by strand invasion and formation of a P-loop has been used in combination with oligodeoxyribonucleotides or fluorescent probes for the capture of double-strand DNA or the detection of target sequences by rolling circle DNA amplification. $^{33,34}$ 
Solid-phase hybridization techniques

PNAs can be used in many of the same hybridization applications as natural or synthetic DNA probes but with the added advantages of tighter binding and higher specificity. Their neutral backbone significantly increases the rate of hybridization in assays where either the target or the probe is immobilized. This could lead to faster and easier procedures in most standard hybridization techniques, such as Southern and Northern blotting. ${ }^{6}$ An alternative to Southern analysis is also the PNA pregel hybridization process, which significantly simplifies the procedure of Southern hybridization. ${ }^{35}$ Labelled PNAs are then used as probes, allowing hybridization to a denatured dsDNA sample at low ionic strength prior to loading on the gel. This is different from conventional Southern blotting where hybridization occurs after gel electrophoresis and membrane transfer. Here, the mixture is directly subjected to electrophoresis for separation of bound and unbound PNA probes. Owing to their neutral charge, excess unbound PNA probes do not migrate in an electrical field. The PNA-DNA hybrids are then blotted onto a nylon membrane and detected using standard chemiluminescent techniques. The method is sensitive enough to detect a single mismatch in a DNA sample.

Another gel-based strategy, known as affinity electrophoresis, can be exploited with PNA probes. In this case, the slowdown of DNA during electrophoretic passage is related to its hybridization with a complementary PNA probe entrapped in the gel matrix. Likewise, hybridization PNA-based biosensor procedures have been developed in which a single-stranded PNA probe is immobilized onto optical or mass-sensitive transducers to detect the complementary strand or corresponding mismatch in a DNA sample solution. The hybridization events are converted in electric signals by the transducers. Jensen et $a l^{3}$ reported the real-time analysis of the kinetics of PNA-DNA and PNA-RNA hybridizations using the BIAcore (biomolecular interaction analysis) technique. Also, MALDI-TOF mass spectrometry was successfully used with mass-labelled PNA probes for the detection of single-nucleotide polymorphisms. $^{36}$

Owing to their remarkable sequence discrimination, PNAs also have great potential in the growing area of whole genome analysis. Weiler et $a l^{37}$ demonstrated that PNA oligomers may be efficiently incorporated into microarrays, and recently automatic production of PNA library arrays has been reported. ${ }^{38}$

\section{In situ hybridization (PNA-FISH)}

The efficiency of PNAs as hybridization probes has also been demonstrated in fluorescence in situ hybridization (FISH) applications. Thank to its high binding specificity, a single 15-mer PNA probe can substitute to a set of longer DNA probe. Also, the neutral backbone of PNAs allows them to bind to DNA or RNA under low ionic strength conditions, which discourage reannealing of complementary genomic strands. This is particularly advantageous for in situ targetting of repeat sequences for which both the length and the repetitive nature can favour renaturation over hybridization with probes. Additional benefits of using PNAs in situ are lower background signals, mild washing procedure and unlimited stability of the probe mixture. ${ }^{39}$ Using the recommended procedure for in situ labelling and washing, no significant residual signals were seen, and the background signals remain low probably because of the high binding specificity of the PNA probes, which limits the unlegitimate binding of probes, and the short time of in situ hybridization. PNA probes are compatible with a wide range of reporter molecules and fluorochromes including fluoresceine, rhodamine as well as cyanine and Alex dyes available in a large variety of colours.

The PNA-FISH technique was first developed for quantitative telomere analysis. Using a unique fluoresceinlabelled PNA probe, Lansdorp et al ${ }^{40}$ performed the in situ labelling of human telomeric repeat sequences and the data obtained allowed accurate estimates of telomere lengths. Subsequently, telomeric PNA probes were used in several in situ studies of cancer and ageing. ${ }^{41,42}$ Further developments were focused on the in situ specific identification of human chromosomes on both metaphases and interphase nuclei, using PNA probes specific for satellite repeat sequences of various chromosomes (chromosomes $1,2,7,9,11,17,18, \mathrm{X}$ and $\mathrm{Y})$. Multicolour PNA experiments were thus reported on lymphocytes, amniocytes and fibroblasts from normal subjects and patients with numerical abnormalities. ${ }^{43,44}$ Chen et al ${ }^{45}$ reported that PNA probes could discriminate in situ between two centromeric DNA repeats that differ by only a single base pair. Similar results were obtained with PRINS primers, oligonucleotide probes or padlock probes, ${ }^{32,46,47}$ but never with standard FISH probes. The identification of chromosomal variations and the analysis of polymorphisms could greatly benefit from the discrimination power of PNAs. The flexible procedure of PNA synthesis allows to consider the further creation of allele-specific PNA probes.

Recently, PNA-FISH has been adapted to in situ chromosomal analysis of the human sperm. ${ }^{48}$ This adaptation constituted an interesting challenge because of the particularities of human spermatozoa nuclei in terms of genomic compaction and accessibility of DNA sequences. Comparative estimates of disomies $\mathrm{X}, \mathrm{Y}$ and 1 were performed in sperm from healthy subjects using FISH, PRINS and PNA procedures in parallel. An equivalent quality of in situ nuclear labelling and similar results were obtained with the three methods. However, the hybridization timing of PNA probes (ie $45 \mathrm{~min}$ ) appeared to be significantly shortened in comparison with FISH reaction on sperm, which requires an overnight hybridization in order to be efficiently completed. The fast hybridization 
kinetics of PNA was similar to the kinetics of PRINS reaction. The similarity between PNA and PRINS might be essentially due to the small size of both PNA probes and PRINS primers, which do not exceed 30 bases in length. These data point out the importance of the probe size for in situ assays and consequently the great potentiality of the PNA approach, since high specific binding can be obtained with a short, unique PNA oligomer. This study also demonstrates the efficiency of the multicolour PNA procedure and shows that PNA could be a powerful alternative to FISH and PRINS for in situ chromosomal investigations. The application of PNA in cytogenetics opens up the possibility of multiplex assays using various fluorophores or combinations of PNA probes with DNA probes or PRINS primers.

\section{Conclusion}

Since the first description of PNAs, various types of applications have been reported, demonstrating the great interest of the PNAs for biotechnology and molecular genetics. Powerful applications of PNA have also emerged in microbiology, virology and parasitology. PNA-based applications benefit from the unique physico-chemical properties of PNA molecules, enabling development of simple and robust assays in molecular genetics and cytogenetics. PNAs are finding increasing uses as probes and other tools in genetics, because these molecules exhibit enhanced binding afinity, increased stability and resistance in biological fluids and reduced nonspecific effects. As pointed out by Nielsen and Egholm $^{6}$ 'the greatest contribution of PNAs may come from the development of new applications that cannot be performed using oligonucleotides'. New chemical modifications of the original PNA backbone may contribute to increasing the potentialities of PNAs and lead to the development of novel applications and PNA-dependent projects in many areas of biology and therapy.

\section{Acknowledgements}

This work was supported by an European grant COPERNICUS 2 (Contract ICA-CT-2000-10012, proposal ICA2-1999-20007).

\section{References}

1 Nielsen PE, Egholm M, Berg RH, Buchardt O: Sequence-selective recognition of DNA by strand displacement with a thyminesubstituted polyamide. Science 1991; 254: 1497-1500.

2 Koch T, Naesby M, Wittung P et al: PNA-peptide chimera. Tetrahedron Lett 1995; 36: 6933-6936.

3 Jensen KK, Orum H, Nielsen PE, Norden B: Hybridization kinetics of peptide nucleic acids (PNA) with DNA and RNA studied with BIAcore technique. Biochemistry 1997; 36: 5072-5077.

4 Demidov V, Frank-Kamenetskii MD, Egholm M, Buchard O, Nielsen PE: Sequence selective double strand DNA cleavage by peptide nucleic acid (PNA) targeting using nuclease S1. Nucl Acids Res 1993; 21: 2103-2107.
5 Rasmussen H, Kastrup JS, Nielsen JN, Nielsen JM, Nielsen PE: Crystal structure of a peptide nucleic acid (PNA) duplex at $1.7 \mathrm{~A}$ resolution. Nat Struct Biol 1997; 4: 98-101.

6 Nielsen PE, Egholm M: An introduction to peptide nucleic acid. Curr Issues Mol Biol 1999; 1: 89-104.

7 Giesen U, Kleider W, Berding C, Geiger A, Orum H, Nielsen PE: A formula for thermal stability $\left(T_{\mathrm{m}}\right)$ prediction of PNA/DNA duplexes. Nucl Acids Res 1998; 26: 5004-5006.

8 Boffa LC, Morris PL, Carpeneto EM, Louissaint M, Allfrey VG: Invasion of the CAG triplet repeats by a complementary peptide nucleic acid inhibits transcription of the androgen receptor and TATA binding protein genes and correlates with refolding of an active nucleosome containing a unique AR gene sequence. J Biol Chem 1996; 271: 13228-13233.

9 Vickers TA, Griffity MC, Ramasamy K, Risen LM, Freier SM: Inhibition of NF-kappaB spécifique transcriptional activation by PNA stand invasion. Nucleic Acids Res 1995; 23: 3003-3008.

10 Nielsen PE, Egholm $M$, Buchardt $O$ : Sequence specific transcription arrest by PNA bound to the template strand. Gene 1994; 149: 139-145.

11 Mischiati C, Borgatti M, Bianchi N et al: Interaction of the human NF-kappaB p52 transcription facture with DNA-PNA hybrides mimicking the NF-kappaB binding sites of the human immunodeficience virus type 1 promoter. J Biol Chem 1999; 274: 33114-33122.

12 Knudsen H, Nielsen PE: Antisense properties of duplex- and triplex-forming PNAs. Nucleic Acids Res 1996; 24: 494-500.

13 Hanvey JC, Peffer NJ, Bisi JE et al: Antisense and antigene properties of peptide nucleic acids. Science 1992; 258: 1481-1485.

14 Mologni L, Lecoutre P, Nielsen PE, Gambacorti-Passerini C: Additive antisense effects of different PNAs on the in vitro translation of the PML/RARalpha gene. Nucleic Acids Res 1998; 26: $1934-1938$

15 Doyle DF, Braasch DA, Simmons CG, Jaowski BA, Corey DR: Inhibition of gene expression inside cells bypeptide nucleic acids: effect of mRNA target sequence, mismatched bases, and PNA length. Biochemistry 2001; 40: 53-64.

16 Cutrona G, Carpaneto EM, Ulivi M et al: Effects in live cells of a cmyc anti-gene PNA linked to a nuclear localization signal. Nat Biotechnol 2000; 18: 300-303.

17 Pooga $\mathrm{H}$, Soomets $\mathrm{U}$, Hällbrink $\mathrm{M}$ et al: Cell penetrating PNA constructs regulate galanin receptor levels and modify pain transmission in vivo. Nat Biotechnol 1998; 16: 857-861.

18 Fraser GL, Holmgren J, Clarke PB, Wahlestedt C: Antisense inhibition of delta-opioid receptor gene function in vivo by peptide nucleic acids. Mol Pharmacol 2000; 57: 725-731.

19 Nastruzzi C, Cortesi R, Esposito E et al: Liposomes as carriers for DNA-PNA hybrids. J Control Release 2000; 68: 237-249.

20 Norton JC, Piatyszek MA, Wright WE, Shay JW, Corey DR: Inhibition of human telomerase activity by peptide nucleic acids. Nat Biotechnol 1996; 14: 615-618.

21 Wilson GL, Dean BS, Wang G, Dean DA: Nuclear import of plasmid DNA in digitonin-permiabilized cells requires both cytoplasmic factors and specific DNA sequences. I Biol Chem 1999; 274: 22025-22032.

22 Braden LJ, Mohamed AI, Smith CIE: A peptide nucleic acidnuclear localization signal fusion that mediates nuclear transport of DNA. Nat Biotechnol 1999; 17: 784-787.

23 Nielsen PE, Egholm M, Berg RH, Buchardt O: Peptide nucleic acids (PNAs): potential antisense and antigene agents. AntiCancer Drug Des 1993; 8: 53-63.

24 Misra HS, Pandey PK, Modak MJ, Vinayak R, Pandey VN: Polyamide nucleic acid-DNA chimera lacking the phosphate backbone are novel primers for polymerase reaction catalyzed by DNA polymerases. Biochemistry 1998; 37: 1917-1925.

25 Orum H, Nielsen PE, Egholm M, Berg RH, Buchardt O, Stanley C: Single base pair mutation analysis by PNA directed PCR clamping. Nucleic Acids Res 1993; 21: 5332-5336.

26 Cochet O, Martin E, Fridman WH, Teillaud JL: Selective PCR amplification of functional immunoglobulin light chain from 
hybridoma containing the aberrant MOPC 21-derived Vk by PNA-mediated PCR clamping. Biotechniques 1999; 26: 818-822.

27 Fiandaca MJ, Hyldig-Nielsen JJ, Gildae BD, Coull JM: Selfreporting PNA/DNA primers for PCR analysis. Genome Res 2001; 11: $609-613$.

28 Svanvik N, Westman G, Wang D, Kubista M: Light-up probes: thiazole orange-conjugated peptide nucleic acid for detection of target nucleic acid in homogeneous solution. Anal Biochem 2000; 281: 26-35.

29 Seitz O: Solid-phase synthesis of doubly labeled peptide nucleic acids as probes for the real-time detection of hybridization. Angew Chem Int Edn 2000; 39: 3249-3252.

30 Isacsson J, Cao H, Ohlsson L et al: Rapid and specific detection of PCR products using light-up probes. Mol Cell Probes 2000; 14: $321-328$.

31 Bukanov NO, Demidov VV, Nielsen PE, Frank-Kamenetskii MD: PD-loop: a complex of duplex DNA with an oligonucleotide. Proc Natl Acad Sci USA 1998; 95: 5516-5520.

32 Nilson M, Krejci K, Koch J, Kwiatkowski M, Gustavsson P, Landegren U: Padlock probes reveal single-nucleotide differences, parent of origin and in situ distribution of centromeric sequences in human chromosomes 13 and 21. Nat Genet 1997; 16: 252-255.

33 Demidov VV, Bukanov NO, Frank-Kamenetskii D: Duplex DNA capture. Curr Issues Mol Biol 2000; 2: 31-35.

34 Kuhn H, Hu Y, Frank-Kamenetskii MD, Demidov VV: Artificial sitespecific DNA-nicking system based on common restriction enzyme assisted by PNA openers. Biochemistry 2003; 42: 4985-4992.

35 Perry-O'Keefe H, Yao XW, Coull JM, Fuchs M, Egholm M: Peptide nucleic acid pre-gel hybridization: an alternative to Southern hybridization. Proc Natl Acad Sci USA 1996; 93: 14670-14675.

36 Ross PL, Lee K, Belgrader P: Discrimination of single-nucleotide polymorphisms in human DNA using peptide nucleic acid probes detected by MALDI-TOF mass spectrometry. Anal Chem 1997; 69: 4197-4202.

37 Weiler J, Gausepohl H, Hauser N, Jensen ON, Hoheisel JD: Hybridisation based DNA screening on peptide nucleic acid (PNA) oligomer arrays. Nucleic Acids Res 1997; 25: 2792-2799.
38 Matysiak S, Reuthner F, Hoheisel JD: automating parallel peptide synthesis for the production of PNA library arrays. Biotechniques 2001; 31: 896-898.

39 Williams B, Stender H, Coull JM: PNA fluorescent in situ hybridization for rapid microbiology and cytogenetic analysis; in Nielsen PE (ed): Peptide nucleic acids; methods and protocols. Totowa, New York: Humana Press Inc.; 2002, pp 181-193.

40 Lansdorp PM, Verwoerd NP, van de Rijke FM et al: Heterogeneity in telomere length of human chromosomes. Hum Mol Genet 1996; 5: $685-691$.

41 Boei JJWA, Vermeulen S, Natarajan AT: Analysis of radiationinduced chromosomal aberrations using telomeric and centromeric PNA probes. Int J Radiat Biol 2000; 76: 163-167.

42 Mathioudakis G, Storb R, McSweeney PA et al: Polyclonal hematopoiesis with variable telomere shortening in human long-term allogeneic marrow graft recipients. Blood 2000; 96: $3991-3994$

43 Chen $\mathrm{C}$, Wu B, Wei T, Egholm M, Strauss WM: Unique chromosome identification and sequence-specific structural analysis with short PNA oligomers. Mamn Genome 2000; 11: 384-391.

44 Taneja KL, Chavez EA, Coull J, Lansdorp PM: Multicolor fluorescence in situ hybridization with peptide nucleic acid probes for enumeration of specific chromosomes in human cells. Genes Chromosomes Cancer 2001; 30: 57-63.

45 Chen C, Hong YK, Ontiveros SD, Egholm M, Strauss WM: Single base discrimination of CENP-B repeats on mouse and human chromosomes with PNA-FISH. Mamm Genome 1999; 10: $13-18$.

46 Pellestor F, Girardet A, Lefort G, Andréo B, Charlieu JP: Rapid in situ detection of chromosome 21 by PRINS technique. Am J Med Genet 1995; 56: 393-397.

47 O'Keefe CL, Warburton PE, Matera AG: Oligonucleotide probes for alpha satellite DNA variants can distinguish homologous chromosomes by FISH. Hum Mol Genet 1996; 5: 1793-1799.

48 Pellestor F, Andréo B, Taneja K, Williams B: PNA on human sperm: a new approach for in situ aneuploidy estimation. Eu J Hum Genet 2003; 11: 337-341. 